\title{
BER Performance Improvement with Joint Angle-Delay-Polarization Estimation of Multipath Channel Parameters
}

\author{
Cristian Tohanean \\ UPB, ITTC \\ Bucarest, Romania \\ Email: tohaneancristian@yahoo.com
}

\author{
José Picheral \\ SUPELEC \\ Dept. of Signal Processing and Electronic Systems \\ 3 rue Juliot-Curie, F-91192 Gif-sur-Yvette, France \\ Email: jose.picheral@supelec.fr
}

\begin{abstract}
In mobile telecommunications, the quality of demodulation is strongly impacted by channel estimation. The Joint Angle, Delay and Polarization Estimation (JADPE) problem is addressed in this paper when a linear uniform array of crossed dipoles is used to measure the signal. The purpose of this paper is to study the high resolution JADPE ESPRIT method for the estimation of the parameters that characterize each path in order to improve channel estimation and demodulation. A complete system simulation (based on TDD-UTRA standard of UMTS) is proposed in order to evaluate performance in terms of channel estimation accuracy and BER. Simulation results show the interest of considering the polarization as a channel parameter in order to increase the performance. Improvement in the path separation and received signal power is obtained and, because of this, channel estimation accuracy and data estimation accuracy are improved as well.
\end{abstract}

\section{INTRODUCTION}

In mobile communications the propagation channel is characterized by multipaths that are made time-varying by mobile terminal movements. Each path of emitted user signal arriving at a base station antenna array can be described by delay, angle of arrival, amplitude (or fading) and polarization state. Usually the time-variation in angle, delay and polarization is slow compared to the amplitude variations so that angle-delaypolarization parameters can be considered quasistatic over long time intervals. As proposed in radiomobile channel model [1], [2] and confirmed by field measurements [3] the multipaths are characterized by different polarization. Taking account for the polarisation diversity is thus expected to improve the channel estimate.

Different approaches to solve the Joint Angle and Delay Estimation (JADE) problem have been proposed in the literature (see e.g., [4], [5] and references therein) and it is shown that subspace based methods like ESPRIT (Estimation of Sinusoidal Parameters by Rotational Invariance Technique) approach provide good performance in a TD-CDMA system for a realistic channel estimation. On the other hand, preliminary experimental investigations in microcell environments [6] have empirically illustrated that polarization diversity is an attractive solution to achieve decorrelated antenna elements and an extensive model for vector-sensor array processing has been proposed by Nehorai in [7]. In addition, Compton in [8] and Zoltowsky in [9] have proposed ESPRIT-based methods for joint angle and polarization estimation. Recently, Manikas has proposed a Joint Angle, Delay and Polarization Estimation (JADPE) method based on the subspace approach MUSIC (MUltiple SIgnal Classification) in [10], but it requires an expensive bi-dimensional search.

In this paper we study the demodulation performance improvement obtained using a computationally efficient method for channel estimation: the JADPE-ESPRIT method proposed by the authors in [11] provides the multipath parameters exploiting the angle-delay-polarization invariance to improve channel estimation accuracy in wideband TDMA systems. This work continues the research of the above-mentioned papers about JADE approach by increasing the complexity of the antenna array using an array of crossed dipoles. In order to show the improvement due to the use of polarization diversity, performance of the method is evaluated in terms of channel estimation accuracy and BER for the overall system when demodulation is performed.

In this work, the channel estimation is based on the following approach. On each time-slot, the discrete space-timepolarization (S-T-P) channel is estimated from the training sequence using classical approach. Assuming the anglesdelays-polarizations constant over $L$ slots, the $L$ S-T-P channel estimates can be used to re-estimate a unique angle-delaypolarization set by constraining the angles-delays-polarizations to be stationary over $L$ slots independently of the fading variation. To solve JADPE problem, we chose 3D-UnitaryESPRIT for its simplicity and capacity to pair delay and angle for each path. The channel estimate is obtained by substituting estimate of the multipath parameters into the multipath channel model.

The paper is organized as follows. Section II introduces the model for signal and multipath propagation channel. Section III describes the channel estimation using the JADPE-ESPRIT method. Simulation results showing performance improvement are discussed in Sect. IV. Finally, Sect. V contains our conclusions. 


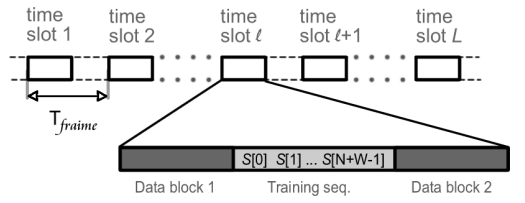

Fig. 1. Frame structure in TD-CDMA system

\section{MODELS}

In the TD-CDMA system the user transmits periodically with an interslot period $T_{\text {frame }}$ as shown in Fig. 1. Each time slot contains the training sequences to estimate the symbol spaced channels.

\section{A. Signal Model}

Assume a digital sequence $s_{k}$ is transmitted over a channel. The response is measured using a uniform linear array consisting of $M$ pairs ( $2 M$ antennas) of crossed dipoles spaced at half wavelength as shown in Fig. 2. The assumption that the receiver's antenna and the transmitter are in the same horizontal plane is made. The base-band signals received by the array of $2 M$ antennas are low-pass filtered and oversampled by $P$ with respect to the chip rate $1 / T$. Assuming that a training sequence is transmitted within each burst, similarly to the signal model in [5], the block of $2 M$ received signals (one for each sensor) within the $\ell$-th time-slot can be compactly written as:

$$
\mathbf{X}(\ell)=\mathbf{H}(\ell) \widetilde{\mathbf{S}}+\mathbf{N}(\ell),
$$

where the columns of the $2 M \times N P$ matrix $\mathbf{X}(\ell)=$ $[\mathbf{x}[0 \mid \ell], \ldots, \mathbf{x}[N P-1 \mid \ell]]$ contain the samples $\mathbf{x}[i \mid \ell]$ received by the $2 M$ antennas at time intervals $T / P, \mathbf{H}(\ell)$ is the $2 M \times W P$ channel matrix where the $m$-th row is the temporal channel for the $m$-th antenna ; the temporal support of the channel is $[0, P W-1] T$ ( $W$ is the channel length in symbole intervals). The convolution matrix $\widetilde{\mathbf{S}}=\left(\mathbf{S} \otimes \mathbf{I}_{P}\right)$ contains the oversampled transmitted sequence $(\mathbf{S}$ is $W \times N$ Toplitz matrix formed with the $N+W$ symbols $s_{k}$ of the transmitted sequence and $\otimes$ denotes the Kroneker product). To avoid ISI between data and training sequence, only $N$ symboles of the received sequence are considered even if the training sequence length is $N+W$ samples. The noise and interference

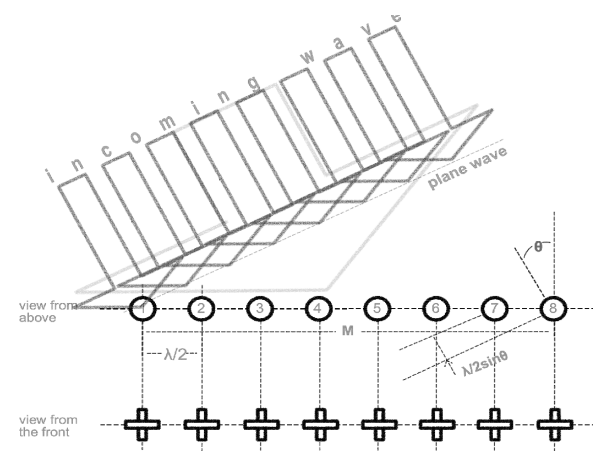

Fig. 2. Uniform linear array structure with crossed dipoles
$\mathbf{N}(\ell)=[\mathbf{n}[0 \mid \ell], \ldots, \mathbf{n}[N P-1 \mid \ell]]$ are assumed Gaussian and non polarized ; they are temporally and spatially uncorrelated.

In TD-CDMA systems, $K$ users transmit simultaneously on the same time-slot. The single-user model (1) can easily be extended to the multi-user case since the received signal at the base station is the sum of the contribution of each user. In the following, for sake of simplicity, only the single-user case will be considered since it has been shown (see [5] and references therein) that the orthogonality of the training sequences allows to reduce the multi-user channel estimation problem to several single-user problems.

\section{B. Multipath Channel Model}

For each time-slot, the $m$-th path can be characterized by the delay $\tau_{m}$, the direction of arrival $\theta_{m}$, the polarization angles $\left(\varepsilon_{m}, \mu_{m}\right)$ and the complex valued slot-varying amplitude (fading) $\beta_{m}(\ell)$. The effects of the mobility of the user and the obstacles in the proximity of the user are that the channel is time-varying. If the user's velocity is not too great, anglesdelays-polarizations are invariant for $L$ time-slots (see [5] [11] and references therein). The amplitudes are assumed constant within the training period (or within the burst) but vary from slot to slot. Fading variations $\beta_{n}(\ell)$ across different slots are a stationary process and the correlation of the faded amplitudes depends on the Doppler frequency.

For the $\ell$-th time-slot, the channel response is an additive combination of $d$ paths:

$$
\mathbf{H}(\ell)=\sum_{m=1}^{d} \beta_{m}(\ell) \mathbf{a}\left(\theta_{m}, \varepsilon_{m}, \mu_{m}\right) \mathbf{g}^{T}\left(\tau_{m}\right),
$$

where the $P W \times 1$ vector $\mathbf{g}\left(\tau_{m}\right)$ represents the known delayed pulse shaping function $g\left(t-\tau_{m}\right)$ sampled at $P / T$ rate. $\mathbf{a}\left(\theta_{m}, \varepsilon_{m}, \mu_{m}\right)$ represents the $2 M \times 1$ vector of the array response to the narrowband signal from the direction $\theta_{m}$ and with the polarization angles $\varepsilon_{m}$ and $\mu_{m}$. For the uniform linear array (see Fig. 2) of omnidirectional antennas spaced half a wavelength apart it is:

$$
\mathbf{a}\left(\theta_{m}, \varepsilon_{m}, \mu_{m}\right)=\mathbf{q}_{m} \otimes \mathbf{v}_{m},
$$

where $\mathbf{q}_{m}=\left[1, \ldots, \varphi_{m}, \varphi_{m}^{M-1}\right]$ is the steering vector with $\varphi_{m}=e^{-j \pi \sin \theta_{m}}$ and $\mathbf{v}_{m}=\left[-\cos \gamma_{m}, \sin \gamma_{m} \cos \theta_{m} e^{j \eta_{m}}\right]$ is the polarization vector expressed in function of angles $\gamma_{m}$ and $\eta_{m}$ (see [8] for details and for the relations between $\varepsilon_{m}, \mu_{m}$ and $\gamma_{m}, \eta_{m}$ ). The polarization ellipse of the electric field of the transverse electromagnetic (TEM) wave propagating can be parameterized by the ellipticity angle $\varepsilon_{m}$ and the orientation (or tilt) angle $\mu_{m}$ as shown in Fig 3.

When applying the vectorization operation (stacking the matrix columns on top of each other) on the $\mathbf{H}(\ell)$ matrix given by (2), we will obtain:

$$
\mathbf{h}(\ell)=\mathbf{U}(\theta, \tau, \varepsilon, \mu) \mathbf{b}(\ell),
$$

where $\mathbf{U}(\theta, \tau, \varepsilon, \mu)=\left[\mathbf{g}\left(\tau_{1}\right) \otimes \mathbf{a}\left(\theta_{1}, \varepsilon_{1}, \mu_{1}\right), \ldots, \mathbf{g}\left(\tau_{d}\right) \otimes\right.$ $\left.\mathbf{a}\left(\theta_{d}, \varepsilon_{d}, \mu_{d}\right)\right]$ is the $2 M W \times d$ matrix of the space-timepolarization manifold and the vector $\mathbf{b}(\ell)=\left[\beta_{1}(\ell), \ldots, \beta_{d}(\ell)\right]^{T}$ 
contains the faded amplitudes. This arrangement decouples the stationary term in the $\mathbf{U}$ matrix and the observation-varying term into the $\mathbf{b}(\ell)$ vector.

\section{ChAnNEL Estimation}

In wideband TD-CDMA systems (e.g. UTRA-TDD standards) the uplink propagation channel (from the mobile to the antenna array) can be assumed to be constant over each time slot, but varying from one time slot to the other. This variation is due to the varying complex fading $\beta_{m}$. However, angles of arrival, delays and polarization are not changing significantly between two slots (see [1], [2], [5] [11] and references therein). In the following, we will assume that these parameters remain constant over $L$ time-slots (where $L<20$ is reasonable for UTRA-TDD standard [5]). Consequently, several observations of the channel can be obtained by considering the bursts transmitted on $L$ time-slots.

\section{A. Maximum Likelihood Estimate}

Assuming that a training sequence is transmitted within each slot, a channel estimation, $\widehat{\mathbf{H}}_{L S}(\ell)$, can be retrieved from the received data using the conventional method. From the signal model (1), assuming that $\mathbf{N}$ is a white Gaussian noise, the Maximum Likelihood (ML) estimate of the channel $\mathbf{H}$ is the Least Square (LS) solution of the equation (1) :

$$
\widehat{\mathbf{H}}_{L S}(\ell)=\mathbf{X}(\ell) \widetilde{\mathbf{S}}^{H}\left(\widetilde{\mathbf{S}} \widetilde{\mathbf{S}}^{H}\right)^{-1} \text {. }
$$

An estimation $\widehat{\mathbf{H}}_{L S}(\ell)$ of the channel (named LS channel estimate in the following) is obtained at each time-slot, this is the conventional channel estimation.

\section{B. Multipath Parameters Estimation}

In order to exploit invariance of some channel parameters, another way to estimate the channel is to use the multipath model (2) proposed in the previous section. This means that the channel estimation is reduced to the estimation of angle, delay, polarization and amplitude for each path. The number of paths needs also to be estimated, classical methods for model order estimation like Akaike Information Criteria can be used to solve this problem.

Assuming that the training sequence is long enough, it can be shown (see [4], [5] and references therein) that it is equivalent to estimate channel parameter from the received signal $\mathbf{X}(\ell)$ or from the LS channel estimate $\widehat{\mathbf{H}}_{L S}(\ell)$. Therefore

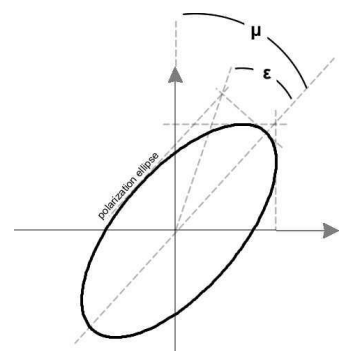

Fig. 3. Definition of polarization parameters $\mu$ and $\varepsilon$ having as a starting point the LS channel estimate, it is possible to use any of the JADPE algorithms [11] or [10].

In this paper, the ESPRIT-like algorithm proposed in [11] is used for joint angle-delay-polarization estimation. This algorithm is based on a subspace approach taking advantage of the invariance properties of the space-time-polarization manifold $\mathbf{U}$ introduced by (4). The JADPE method exploits the principle discussed by Compton in [8] for a separate angle and polarization estimation, the method proposed by Van der Veen in [4] for joint angle and delay estimation. The complete description of the joint estimation using the ESPRIT method is given in [11] with the evaluation of the accuracy of parameter estimation.

Because the standard in discussion is a time division standard, stationary properties can be exploited : the $L$ LS channel estimates $\left\{\widehat{\mathbf{H}}_{L S}(\ell)\right\}_{\ell=1, \ldots, L}$ are collected and considered as $L$ uncorrelated observations of the channel in order to estimate only one set of angle-delay-polarization parameter for the all $L$ slots.

In addition, the use of a joint estimation method provides the angle-delay-polarization estimates $\left(\widehat{\theta}_{m}, \widehat{\tau}_{m}, \widehat{\varepsilon}_{m}, \widehat{\mu}_{m}\right)$ automatically paired for each path. The amplitudes are estimated as the LS solution of (4) where $\mathbf{U}(\theta, \tau, \varepsilon, \mu)$ has been replaced by $\mathbf{U}(\widehat{\theta}, \widehat{\tau}, \widehat{\varepsilon}, \widehat{\mu})$. Each set of estimated parameters is then introduced in (4) to obtain the JADPE channel estimate :

$$
\hat{\mathbf{H}}_{J A D P E}(\ell)=\sum_{m=1}^{d} \hat{\beta}_{m}(\ell) \mathbf{a}\left(\hat{\theta}_{m}, \hat{\varepsilon}_{m}, \hat{\mu}_{m}\right) \mathbf{g}^{T}\left(\hat{\tau}_{m}\right) .
$$

\section{Simulation Results}

The analysis of the performance is based on the data coding used for the UTRA TDD standard of the 3rd generation mobile system. The oversampling factor is $P=2$ with respect to the chip period $(T)$ and $g(t)$ is a square raised cosine with rolloff $22 \%$. The training sequence is periodic with period $N=$ 456 (Burst Type 1). The length of estimated channel is $W=$ 57 chip-spaced samples. We consider a linear array of $M=$ 8 half wavelength spaced antennas. Angle-delay-polarization parameters are supposed to be constant over $L=20$ time slots. The signal to noise ratio is defined for one antenna as $S N R=$ $\sigma_{s}^{2} E_{g} / \sigma^{2}$ where $E_{g}$ is the energy for the chip waveform, $\sigma_{s}^{2}$ and $\sigma^{2}$ are respectively the signal and noise power.

Two different criteria are used to evaluate the system performance: the Mean Squared Error $\left(M S E=\|\mathbf{H}-\widehat{\mathbf{H}}\|^{2} /\|\mathbf{H}\|^{2}\right)$ of the channel estimation and the BER for demodulated sequence after linear decorrelating multiuser detection using the channel estimate [12]. It is important to mention that the errors that affect the two channel estimations (LS and JADPE) are not of the same type : for the LS estimate, the error can be considered as an additive white noise while for the ESPRIT estimate, the error is due to the estimation error of the channel parameters and thus structure error is given by the model (2) . This fact shows that the MSE of the channel estimation is not the best way to judge the quality of the estimation and reveals the necessity of the demodulation test (BER) which will better tells the quality of the estimation. 


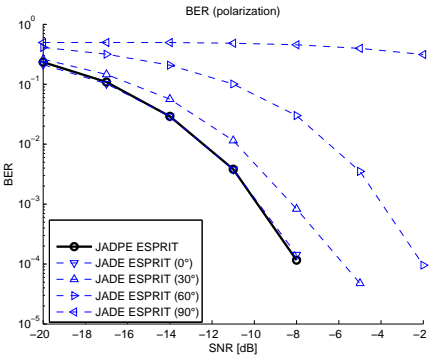

Fig. 4. Comparison of BER for demodulation with JADE and JADPE channe estimate for different polarization tilt angles $(\mu)$ - channel is a 12-path TU with linear polarization.

\section{A. Power Reception Improvement}

The first advantage obtained by taking in consideration the polarization is the power increment of the received signal. In particular, this has the consequence that the channel estimation using crossed dipole arrays has very little dependency on the polarization state while it has dramatic dependency on it for classical arrays.

In general, the polarization of the wave that arrives at the BS antennas is not linear and vertical, simply because the orientation of the mobile antenna is not vertical and/or because of the depolarization of the wave during propagation and reflection. Therefore, in general, there will be a difference between the polarization of the antenna and the polarization of the incoming wave. This difference will lead to a loss of power (antennas capture only a fraction of the incoming wave power). Using crossed dipoles (and thus JADPE ESPRIT estimation) the BS will capture the whole power of the incoming wave. This fact is illustrated by the following test.

BER plotted in Fig 4 are obtained after the demodulation using the estimates obtained with the JADE or JADPE method. The channel model used for simulation is the 12-path channel for typical urban (TU) environment proposed by the COST 207 work group. All the paths have the same polarization parameters, the ellipticity angle is $\varepsilon=0^{\circ}$, and the tilt angle $(\mu)$ takes the values $\left[0^{\circ}, 30^{\circ}, 60^{\circ}, 90^{\circ}\right]$. For this test $L=20$ slots are used. As expected, the performance of the demodulation using the JADPE channel estimate remains the same over the different values of the polarization parameter $\mu$ (due to the use of crossed dipoles) while the performance with the JADE estimate gradually degrades when tilt angle increases.

\section{B. Path Separation Improvement}

The performance improvement obtained in the previous simulations is mainly due to the use of a crossed dipole array. The following tests are showing another progress in data estimation which is the path separation improvement due to joint estimation of all the path parameters.

The test is done for one user using a 2-path channel. Two cases are simulated: in the first situation (named close paths) all the parameters that describes each of the two paths are close in value. So polarization parameters are $\varepsilon=\left[7.5^{\circ}, 15^{\circ}\right]$, $\mu=\left[30^{\circ}, 37.5^{\circ}\right]$, DOA are $\theta=\left[5^{\circ}, 10^{\circ}\right]$ and delays are $\tau=[0.1,0.15] \mu s$ (a difference smaller than $T c / 2$ ); in the second situation (named different paths) the DOA and delays have the same value as in the previous situation but the paths differ by their polarization parameters: $\varepsilon=\left[7.5^{\circ} ; 22.5^{\circ}\right]$, $\mu=\left[30^{\circ} ; 120^{\circ}\right]$. In Fig. 5, on the left-hand side the channel estimation error is plotted for the two methods (LS and JADPE ESPRIT) for both situations (close paths in dashed line and path with different polarization in solid line). For comparison, performance of method without polarization (JADE and LS) are also ploted (dot-dash line). These results first demonstrate the interest of the multipath parameter estimation (JADPE, JADE) with respect to the conventional approach (LS). Comparison of JADE and JADPE methods shows that the path separation improvement due to polarization diversity is about $4 \mathrm{~dB}$ in the case of the path with different polarization and only $1.5 \mathrm{~dB}$ when polarization parameters are close. In addition, these MSE curves show the expected asymptotic behavior of the methods.

In Fig. 5, the curves in the right-hand side show the BER evaluated after demodulation using the true channel and the channel estimates with JADPE and LS methods. The improvement is of about $3 \mathrm{~dB}$ in favor of the different polarization case for the JADPE channel estimation. This test proves the fact that even though the two paths are "close" (parameters values) in delays and angles, by also estimating the polarization of the wave, a better path separation is obtained and thus the channel estimation and the data estimation is improved. Notice also that demodulation with JADPE channel estimate achieves the same performance that demodulation with the true channel.

\section{A Realistic Channel Model}

The last test is done for a realistic situation. The 12 path TU channel [2] is used for two situations: $L=2$ slots and $L=20$ slots (see Fig. 6). The MSE of the channel plotted on the left-side shows that the JADPE method outperforms the JADE one with an approximate gain of $3 \mathrm{~dB}$. It can be observed that the 20 slots are needed to fully exploit the polarization diversity. The figure on the right-hand side shows the performance, in terms of BER: the demodulation using JADPE channel estimate attains almost the same performance as that using the true channel. The difference is less $2 \mathrm{~dB}$ with respect to the true channel and around $5 \mathrm{~dB}$ with respect to the classic approach (LS channel estimate).

\section{CONCLUSIONS}

In this work, the JADPE method has been used for channel parameter estimation in a TD-CDMA system and the performance of the proposed method was tested for the overall system (including demodulation scheme). This paper shows the performance improvement in terms of MSE of channel estimation and BER by introducing the third dimension (polarization) in the received signal processing. Compared to the classic method for channel estimation (LS) and to the existing JADE estimation [5] the tests done on the realistic COST 207 TU 12 path channel showed the performance improvement 

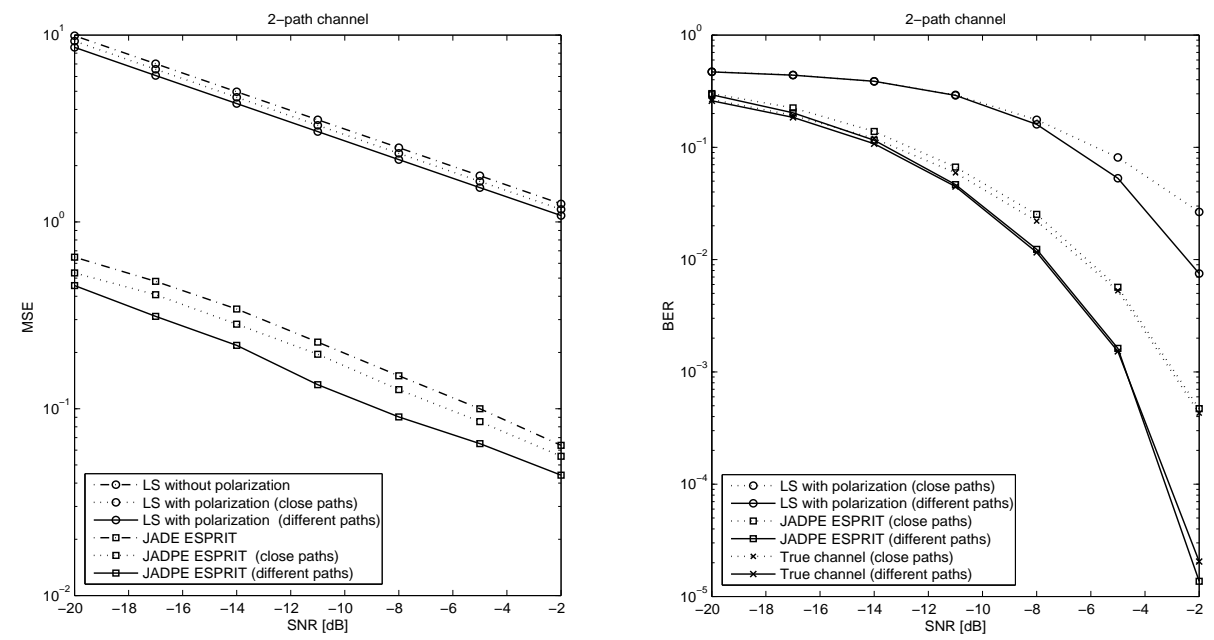

Fig. 5. Path separation : 2-paths channel with close path parameters (dashed line) and with close angle-delay parameters but different polarization (solid line). On the left-hand side the channel estimation error for the 3 methods (LS, JADE and JADPE). On the right-hand side the BER obtained using the true channel and the channel estimates (LS and JADPE) for demodulation.
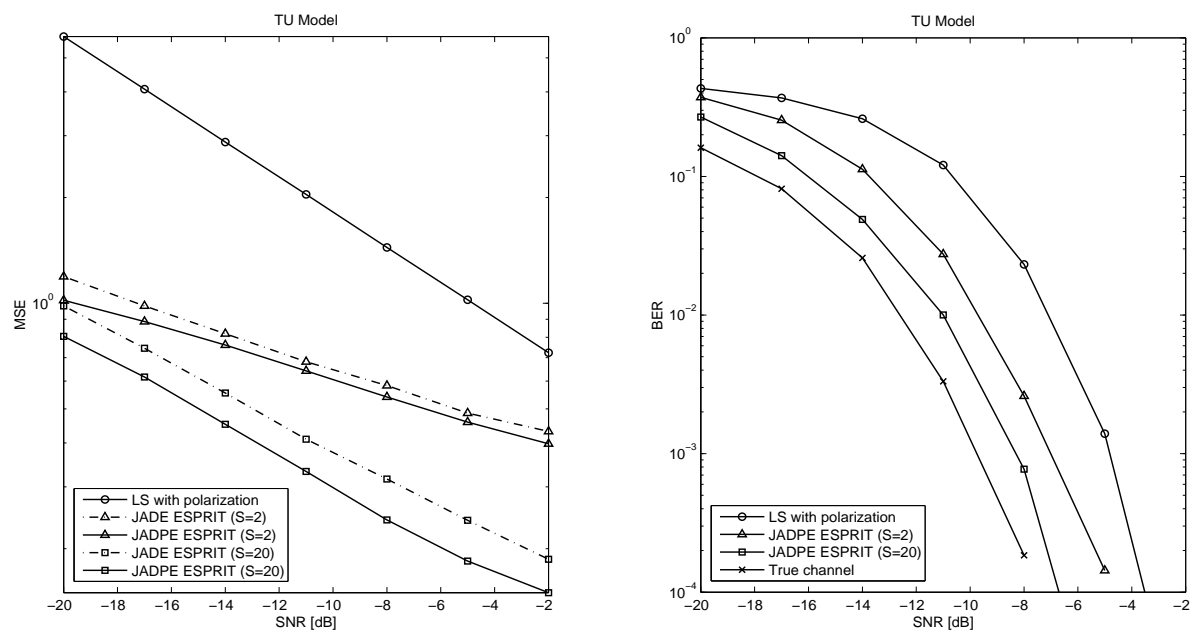

Fig. 6. Performance for 12-path TU Model : on the left-hand side, the channel estimation error for the different methods (LS, JADE $S=2$, JADE $S=20$, JADPE $S=2$ and JADPE $S=20$ ). On the right-hand side the BER obtained using the true channel and the channel estimates for demodulation.

provided by the JADPE ESPRIT channel estimation. Simulations have highlighted the interest of using the polarization diversity: a gain in the received signal power and a better path separation which lead to a better channel estimation and better results in data demodulation.

\section{REFERENCES}

[1] Preben E. Mogensen Klaus I. Pedersen and Bernard H. Fleury, ”Dualpolarized model of outdoor propagation environments for adaptive antennas," 1999, pp. 990-995, IEEE Vehicular Technology Conference.

[2] 3GPP2 3GPP, "Spatial channel model text description," Tech. Rep. SCM111, EURO-COST, Jan. 2003.

[3] K. Kalliola A.F. Molisch P. Vainikainen E. Bonek M. Toeltsch, J. Laurila, "Statistical characterization of urban spatial radio channels," IEEE J. Select. Areas Commun., vol. 20, pp. 539-549, Apr. 2002.

[4] A. Van der Veen M.C. Vanderveen and A. Paulraj, "Estimation of multipath parameters in wireless communications," IEEE Trans. Signal Processing, vol. 46, pp. 682-690, Mar. 1998.

[5] J. Picheral and U. Spagnolini "Angle and Delay Estimation of SpaceTime Channels for TD-CDMA Systems" IEEE Transactions on Wireless Communications, vol. 3, no. 3, May 2004.
[6] J.P. Kermoal, P. Mogensen, S.H. Jensen, J. Bach Andersen, F. Frederiksen, T.B. Srensen, K.I. Pedersen, "Experimental Investigation of Multipath Richness for Multi-Element Transmit and Receive Antenna Arrays", IEEE Proc. 51th VTC, pp. 2004-2008, Tokyo, Japan, May 2000.

[7] A. Nehorai and E. Paldi, "Vector-sensor array processing for electromagnetic source localization," IEEE Trans. Signal Processing, vol. 42, pp. 376-398, Feb. 1994

[8] J. Li and R. T. Compton, "Angle and polarization estimation using rotation invariance with a polarization sensitive array," IEEE. Trans. Antennas Propag., vol. 39, pp. 1376-1383, Sept. 1991.

[9] M. D. Zoltowoski and K. T. Wong, "ESPRIT-based 2-d direction finding with a sparse uniform array of electromagnetic vector sensor," IEEE Trans. Signal Processing, vol. 48, pp. 2195-2204, Aug. 2000.

[10] W.P. Jason and A. Manikas, "Diversely polarised arrays in DS-CDMA: a space-time channel estimation approach", May 2002, vol. 3, pp. 13-17, ICASSP, Orlando, FL, USA.

[11] J. Picheral and F. Piqueres, "Joint Angle-Delay-Polarisation estimationby Esprit-Like Method for Multipath Channel Identification", ESIPCO, Vienne, Autria, Sept. 2004.

[12] P. Jung and J. Blanz, "Joint detection with coherent receiver antenna diversity in CDMA mobile radio systems," IEEE Trans. Vehic. Technol., vol. 44, pp. 76-88, Feb. 1995. 